\title{
Epidural Hematoma Presenting with Severe Neck Pain without Neurological Deficit - A Late Complication of Posterior Cervical Spine Surgery: Presentation of Three Unusual Cases
}

\author{
Mustafa H. Khan, Joon Y. Lee, William F. Donaldson III, James D. Kang \\ Department of Orthopedic Surgery, University of Pittsburgh Medical Center, \\ Pittsburgh, PA 15213, USA
}

\begin{abstract}
Postoperative epidural hematoma (EDH) usually present with neurological deficit. Massive EDH presenting with only severe pain without neurological deficit are rare. Atypical presentations of postoperative EDHs may lead to delayed diagnosis and treatment. We present three such cases after posterior cervical spine surgery. Three patients presented with severe neck pain and spasms without motor deficits several days after posterior cervical decompressive procedures. Imaging studies identified compressive EDHs at the surgical site with severe compression of the spinal cord. All were treated with emergent decompression, with resulting improvement of symptoms and pain relief without further neurological sequelae. In conclusion, postoperative EDHs after posterior cervical spine surgery may result in minimal neurological deficit. Our report reminds surgeons to keep this possibility in mind when patients complain of unusually severe neck pain and spasms after posterior cervical spine surgery.
\end{abstract}

Key Words: Cervical spine, Epidural hematoma, Postoperative complication

\section{Introduction}

Postoperative neurological deficit after a decompressive cervical spine surgery is a rare but devastating complication $^{1,2}$. One rare cause of such complication is the formation of a postoperative epidural hematoma $(\mathrm{EDH})^{3}$. If detected early, surgical evacuation of the EDH and decompression of the spinal cord can result in marked neurological improvement ${ }^{4,5}$. A delay in treatment may lead to persistent neurological impairments.

Most commonly, post-operative EDH presents with rapid neurological deterioration soon after the index procedure ${ }^{6,7}$. In this report, we describe three atypical cases of postoperative EDHs where marked compression of the cervical spinal cord resulted in severe neck pain and spasms with no initial motor dysfunction.

\section{Case Reports}

\section{Case 1}

Clinical presentation: A 55-year old male with cervical myelopathy underwent C3-7 laminectomy and instrumented fusion with lateral-mass screws and rods. The patient had an unremarkable hospital course with no new neurological deficits, and he was discharged to home three days after the procedure. Nine days postoperatively he presented to the emergency room with complaints of progressively increasing severe neck pain and muscle spasms with mild paresthesias radiating down to the fingertips. He denied any gait abnormalities, upper or lower extremity weakness or any bowel/ bladder changes. On examination he had severe neck pain with any attempted motion and severe tenderness to palpation over the posterior neck incision. Otherwise he

Corresponding author: James D. Kang, MD

Division of Spinal Surgery, Department of Orthopedic Surgery, University of Pittsburgh Medical Center

3741 Fifth Avenue, Suite 1010, Pittsburgh, PA 15213, USA

Tel: +82-412-605-3241, Fax: +82-412-687-3724, E-mail: kangjd@upmc.edu 
had 5/5 muscle strength in all muscle groups in upper and lower extremities bilaterally.

Radiological findings: An urgent MRI was obtained which showed a large, expansive hematoma that was compressing the spinal cord posteriorly from C3-7 (Fig. 1A). Axial images showed that the spinal cord was severely compressed (Fig. 1B).

Diagnosis and treatment: A diagnosis of epidural hematoma was made. The patient was taken emergently to the operating room. Intraoperative findings revealed a large subfascial organized hematoma that was carefully evacuated. After performing a thorough irrigation, the wound was examined for excessive bleeding. No active bleeding was found. A drain was placed in the subfascial space, the fascia was closed followed by skin closure.

Outcome: Post-operatively, his pain and parasthesia completely resolved, and he was discharged from the hospital without any further sequalae.

\section{Case 2}

Clinical presentation: A 58-year old female with a history of cervical stenosis and myelopathy underwent an uncomplicated C3-7 laminoplasty. On the second postoperative day, she complained of gradually increasing neck pain and spasms. She had 5/5 strength throughout all extremities, and no bowel/ bladder dysfunction. She stated that her neck pain and spasms were worse with sitting up, and the symptoms were somewhat relieved when she was positioned supine in bed. Her symptoms did not improve over the next three days despite medical treatment with muscle relaxants for presumed muscle spasms. Finally, a MRI scan was ordered to evaluate the cervical spine for possible cord compression from an EDH.

Radiological findings: The MRI showed a large posterior EDH that was compressing the spinal cord (Fig. 2). Some of the hematoma had tracked under the lamina of T1 near the T1-2 area.

Diagnosis and treatment: The patient was taken emer-

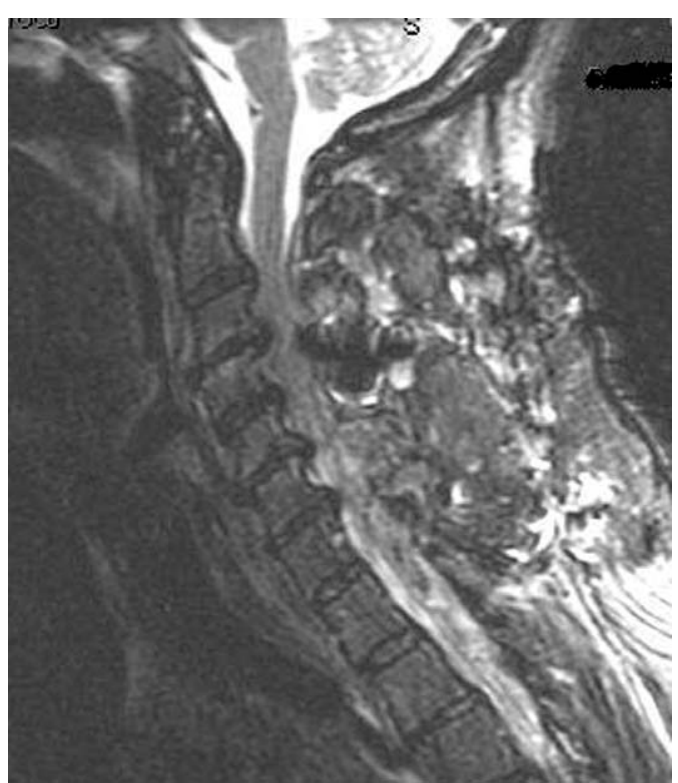

Fig. 2. Sagittal MRI showing large hematoma compressing the spinal cord from C3-T1.

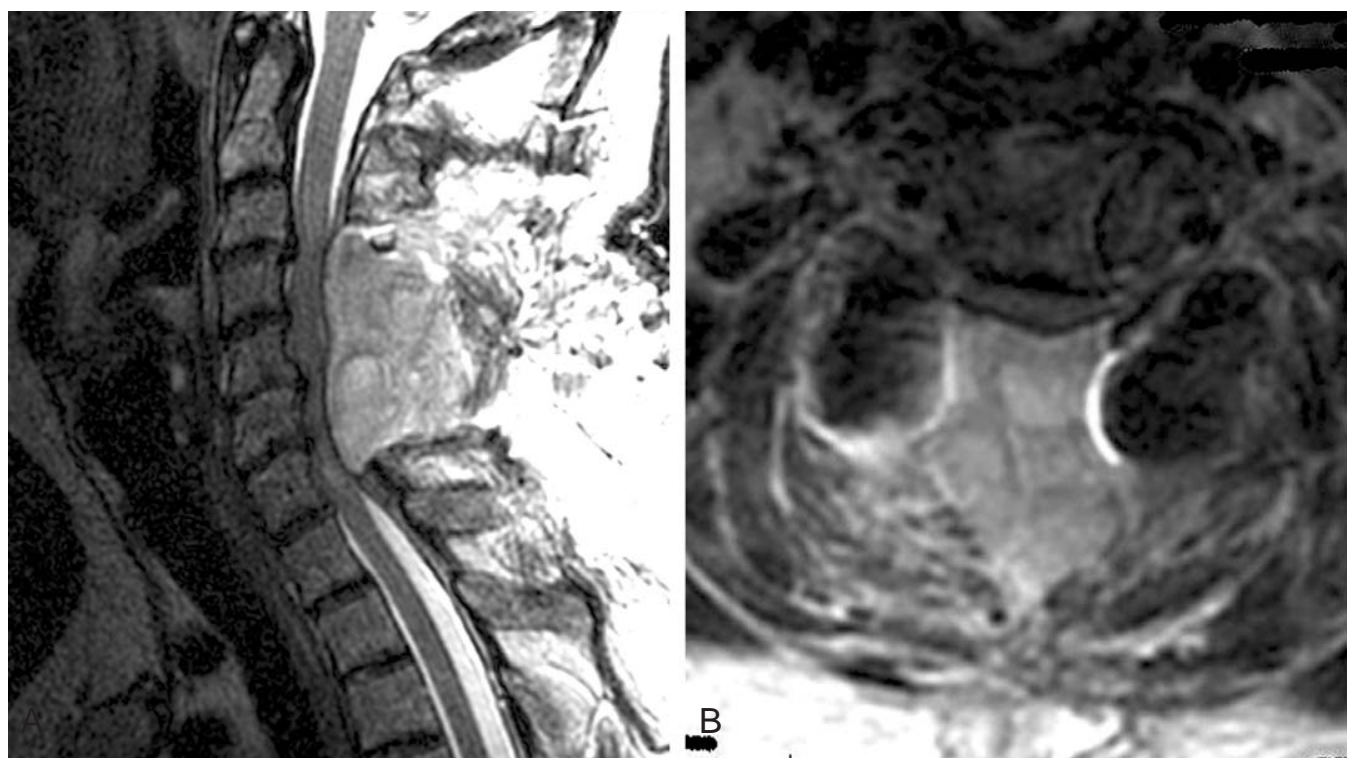

Fig. 1. (A) Sagittal MRI showing large hematoma compressing the spinal cord from C3-C7. (B) Axial MRI imaging showing a flattened spinal cord. 
gently to the operating room for irrigation and debridement, evacuation of the EDH. Intraoperatively, a large, well-organized jelly-like posterior cervical hematoma was noted in the epidural space, which was decompressed by thoroughly evacuating the hematoma, and a T1 laminectomy was performed to evacuate the hematoma under the T1 lamina. The laminoplasty door was noted to be wide open and in excellent position. There were no SSEP changes throughout the procedure.

Outcome: The patient woke up from the procedure neurologically intact. Her neck symptoms had completely resolved, and she did not have any motor/ sensory sequelae. She was discharged to a rehab facility without further complications.

\section{Case 3}

Clinical presentation: The patient was a 66-year old female who underwent a C3-7 laminectomy and fusion with instrumentation without any complications. The second day after the operation the patient started having mild neck pain and spasms without any focal neurological deficits. She was given a brief course of intravenous decadron, and the symptoms resolved completely. She was discharged home in a stable condition, neurologically intact. Over the next 24 hours, the neck spasms and pain returned, causing her to return to the emergency room for evaluation. There were no motor deficits, no bowel and bladder changes. Since an EDH was clinically suspected, an urgent cervical spine MRI was obtained.
Radiological findings: The MRI showed a large posterior EDH that was compressing the spinal cord in the C4-5 region (Fig. 3A and B).

Diagnosis and treatment: The patient was taken emergently to the operating room for urgent decompression of the EDH. A large hematoma, found to be compressing the spinal cord, was evacuated.

Outcome: At the completion of the procedure the patient woke up without any complications. The neck pain and spasms had completely resolved. Subsequently she had an uneventful postoperative course.

\section{Discussion}

Epidural hematomas typically present with neurological deficit such as motor weakness or bowel/ bladder dysfunction. Most often such hematomas manifest themselves in the immediate postoperative period when the patient starts to complain of a sudden inability to move the extremities ${ }^{1,6,7}$. However, as we have demonstrated, atypical presentation of a postoperative EDH is possible which may result in a delay of diagnosis.

The cases illustrated in this report are unusual for two reasons. First, these patients had a slowly progressive hematoma that accumulated over several days, with atypical symptoms. Secondly, pain (rather than motor weakness) was the primary complaint. All three patients complained of severe 'neck spasms' instead of motor weakness. Due to these reasons the appropriate diagnosis was delayed. Once
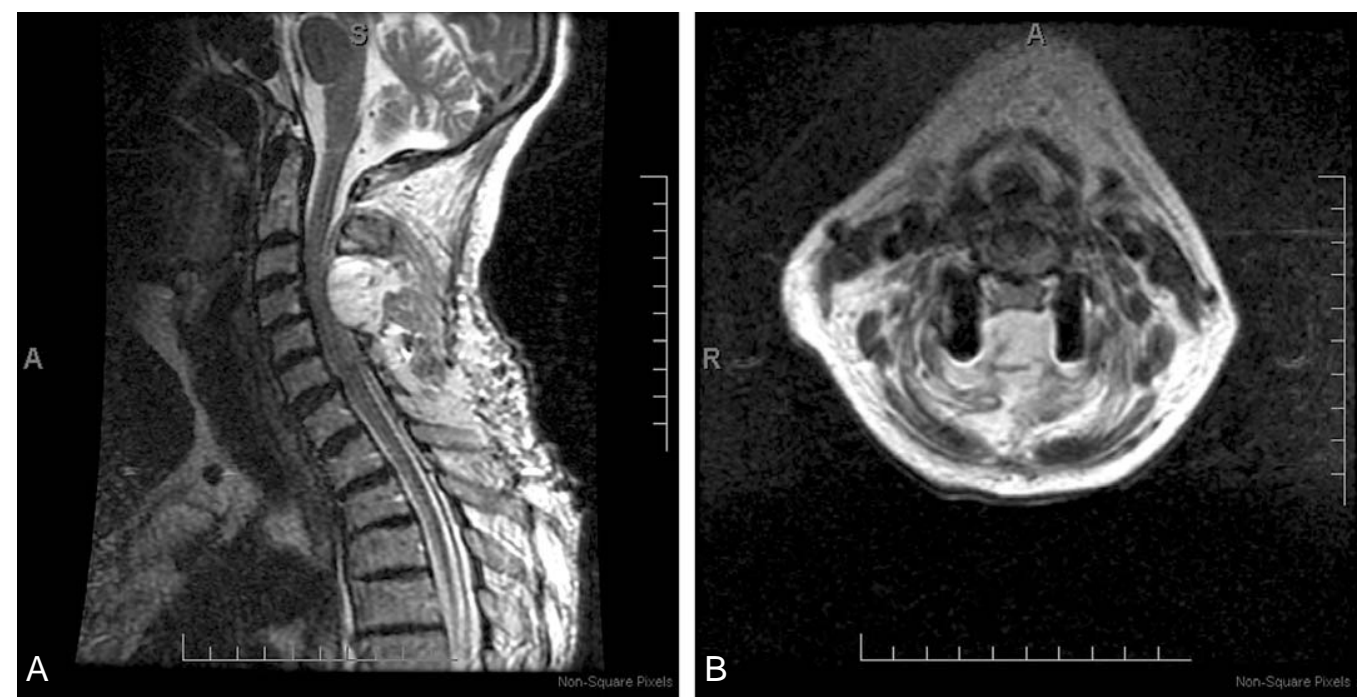

Fig. 3. (A) Sagittal MRI showing large hematoma compressing the spinal cord from C4-5. (B) Axial MRI imaging showing a flattened spinal cord. 
an EDH was diagnosed, prompt intervention was initiated and all three patients experienced a sudden relief of symptoms after the evacuation of the offending hematoma. As to why these patients did not have objective motor weakness despite severe cord compression, we speculate that the slow, progressive collection of blood over a period of several days allowed the spinal cord to adapt to this compression. However, if such a severe compression was to occur in the immediate postoperative period, an acute motor weakness would almost certainly be expected ${ }^{5}$. As shown by other authors, we have also demonstrated that urgent evacuation of the EDH led to an immediate resolution of symptoms?

Our report serves to remind the surgeon that it is possible for a patient to develop a slowly-evolving epidural hematoma over a period of several days after cervical spine surgery. Normal motor function does not by itself rule out the possibility of an EDH. Neck pain out of proportion to the normal post-operative recovery should be investigated further, including an imaging study such as an MRI to look for post-operative EDH. Prompt diagnosis is essential so that the appropriate intervention can be initiated.

\section{Conclusions}

Early recognition and treatment of postoperative EDH is essential. Evacuation of the hematoma and decompression can lead to improvements in neurological function and pain. In rare cases, a slow-growing postoperative EDH may be manifested by severe pain out of proportion to the physical exam in the presence of normal motor function. Therefore, a normal motor exam should not lead the surgeon to rule out a possible diagnosis of an $\mathrm{EDH}$.

\section{REFERENCES}

1. Freedman BA, Potter BK, Kuklo TR: Managing neurologic complications in cervical spine surgery. Curr Opin Orthop 2005; 16: 169-177.

2. Bhardwaj A, Long DM, Ducker TB, Toung TJ: Neurologic deficits after cervical laminectomy in the prone position. J Neurosurg Anesthesiol 2001; 13: 314-319.

3. Binder DK, Sonne DC, Lawton MT: Spinal epidural hematoma. Neurosurg Q 2004; 14: 51-59.

4. Kou J, Fischgrund J, Biddinger A, Herkowitz H: Risk factors for spinal epidural hematoma after spinal surgery. Spine 2002; 27: 1670-1673.

5. Groen RJ, van Alphen HA: Operative treatment of spontaneous spinal epidural hematomas: A study of the factors determining postoperative outcome. Neurosurgery 1996; 39: 494-508.

6. Hans P, Delluze PP, Born JD, Bonhomme V: Epidural hematoma after cervical spine surgery. J Neurosurg Anesthesiol 2003; 15: 282-285

7. Lee JY, Schwartz DM, Anderson DG, Hilibrand AS: Epidural hematoma causing dense paralysis after anterior cervical corpectomy. A report of two cases. J Bone Joint Surg Am 2006; 88: 198-201. 